\title{
Influence of soya lecithin, sorbitan and glyceryl monostearate on physicochemical properties of organogels
}

\begin{abstract}
The objective of this study is to investigate the effects of three different organogelators, sorbitan monostearate (SMS), soya lecithin (SL) and glyceryl monostearate (GMS) prepared at different concentrations $(12 \%, 15 \%$ and $18 \%, \mathrm{w} / \mathrm{w})$ on the structural, thermal and mechanical properties of palm olein (PO)-based organogels. Polarized light microscopy analysis revealed needle-like crystals in SMS-PO, rod-shaped tubules in SL-PO and rosette-like aggregates in GMS-PO organogels. Intermolecular hydrogen bonding and van der Waals forces were the main drivers for the self-aggregation of these organogelators in PO, as observed in Fourier transform infrared (FTIR) spectroscopy. X-ray diffraction (XRD) results indicated $\beta$ '-type polymorphic structure in SL-PO and GMS-PO. As the concentration of organogelators increased, there was a corresponding increase in the firmness, gel-sol transition (Tgs) and melting temperatures of the organogels. SMSPO with amorphous structure had the lowest firmness, thus produced weaker gel with lower thermal stability. The oil binding capacity (OBC) of both SL-PO and GMS-PO were over 90\%, significantly higher than that of SMS-PO organogels. These findings indicate that crystallization is the key determinant factor to the final properties of the organogel networks. This is influenced by the molecular structure and the concentration of the organogelators used.
\end{abstract}

